\title{
LIPSCHITZ SPACES AND MIXED LEBESGUE SPACES
}

\author{
W. R. MADYCH
}

\begin{abstract}
It is shown that translation invariant linear operators which improve Lipschitz classes behave almost as well as the corresponding fractional Riesz transforms when applied to the mixed Lebesgue spaces. These results partially generalize some of the theorems concerning Riesz transforms and mixed Lebesgue classes due to Adams and Bagby, Lizorkin, and others.
\end{abstract}

1. Introduction. It is known that the two variable Riesz transforms of order $\alpha, \alpha>0$, maps $L^{p_{2}}\left(L^{p_{1}}\right)$ into $L^{q_{2}}\left(L^{q_{1}}\right)$ whenever $1<p_{i} \leq q_{i}<\infty, i=1,2$, and $\sum\left(1 / p_{i}-1 / q_{i}\right)=\alpha$; see [1]. In view of the results of E. M. Stein and A. Zygmund, [9], concerning translation invariant operators on Lipschitz spaces and Lebesgue spaces, it seems reasonable to suspect that results analogous to the one quoted above hold for all translation invariant linear operators which map $\operatorname{Lip} \beta$ into $\operatorname{Lip}(\alpha+\beta)$.

In this note we show that, roughly speaking, the above suspicion is well founded.

We use a characterization of the class of operators mentioned above which goes back to Zygmund [11]. However, our results are formulated in terms of Besov spaces, which have been studied by many authors, for example see [7] or [8]. Relevant definitions and facts which we need concerning Besov spaces are contained in $\S 2$. $\S 3$ contains the statement of the main results. Their proofs are contained in $\S 4$. Generic constants are always denoted by the symbol $C$. They need not be the same at different occurrences.

2. Besov spaces and translation invariant operators. Let $\hat{\phi}$ be a nonnegative radial function in $C^{\infty}\left(R^{n}\right)$ with support in $1 \leq|\xi| \leq 2$ and such that $\int_{0}^{\infty} \hat{\phi}(t \xi) d t / t=1$ for all $\xi \neq 0$. The function $\phi$ is the Fourier transform of $\hat{\phi}$ and $\phi_{t}$ is defined by $\phi_{t}(x)=t^{-n} \phi\left(t^{-1} x\right)$. The Schwartz space of rapidly decreasing infinitely differentiable functions on $\mathbf{R}^{n}$ is denoted by $S$ and $S_{0}=\{\psi \in S$ : $\int_{\mathbf{R}^{n}} P(x) \psi(x) d x=0$ for all polynomials $\left.P(x)\right\}$. The dual of $S_{0}$ is denoted by $S_{0}^{\prime}$. Notice that $S_{0}^{\prime}=S^{\prime} / P$, where $P$ is the subspace of polynomials. Also, whenever it makes sense, the convolution of two distributions, $f$ and $g$, is denoted by $f * g$.

Given real numbers $\alpha$ and $p,-\infty<\alpha<\infty$ and $1 \leq p \leq \infty$, the space $\Lambda_{\alpha}^{p}$ is the subspace of $S_{0}^{\prime}$ consisting of those $f$ for which $f * \phi_{t}$ is in $L^{p}\left(\mathbf{R}^{n}\right)$ for all $t>0$ and

$$
\|f\|_{p, \alpha}=\sup _{t>0}\left(t^{-\alpha}\left\|f * \phi_{t}\right\|_{L^{p}}\right)
$$

is finite. $\Lambda_{\alpha}^{p}$ is a Banach space with the norm given by (1).

Received by the editors July 28, 1981.

1980 Mathematics Subject Classification. Primary 42B99, 26 B35.

(C) 1982 American Mathematical Society 0002-9939/81/0000-0218/\$02.50 
There are many equivalent norms on $\Lambda_{\alpha}^{p}$. For example, it is well known that if $\alpha>0$ and $m$ is an integer greater than $\alpha$ then

$$
\sup _{|y|>0}\left(|y|^{-\alpha}\left\|\Delta_{y}^{m} f\right\|_{L^{p}}\right)
$$

is a norm equivalent to (1), where $\Delta_{y}^{m}$ denotes the $m$ th difference operator in $y$, that is, $\Delta_{y} f(x)=f(x+y)-f(x)$ and $\Delta_{y}^{m} f=\Delta_{y} \Delta_{y}^{m-1} f$ if $m \geq 2$. This is the familiar Lipschitz norm. We will not discuss these facts further here. However, it should be mentioned that one can introduce one more parameter into the definition $\Lambda_{\alpha}^{p}$ by replacing the $L^{\infty}$ norm in the $t$ variable with an appropriate $L^{q}$ norm. Namely

$$
\int_{0}^{\infty}\left(t^{-\alpha}\left\|f * \phi_{t}\right\|_{L^{p}}\right)^{q} \frac{d t}{t}
$$

The resulting space is denoted by $\Lambda_{\alpha}^{p, q}$. These classes of spaces are sometimes called Besov spaces and denoted by $B_{\alpha}^{p, q}$.

THEOREM 1. Suppose $f \rightarrow T f$ is a translation invariant linear operation mapping $\Lambda_{\alpha}^{p, q}$ continuously into $\Lambda_{\beta}^{r, s}$. Then there is a unique $k$ in $S_{0}^{\prime}$ such that $T f=k * f$ for all $f$ in $S_{0}$.

THEOREM 2. The transformation $f \rightarrow k * f$ maps $\Lambda_{\alpha}^{\infty}$ continuously into $\Lambda_{\beta}^{\infty}$ if and only if $k$ is in $\Lambda_{\delta}^{1}$ where $\delta=\beta-\alpha$.

With minor modifications the proof of Theorem 1 is the same as that of its analogue in [10]. Theorem 2 is simply a fancy variant of Zygmund's theorem, [11], which we mentioned in the introduction. See [7, p. 135] for a proof of this version.

3. Mixed Lebesgue spaces and $\Lambda_{\alpha}^{p}$. Given $P=\left(p_{1}, \ldots, p_{n}\right), 1 \leq p_{i} \leq \infty$, $i=1, \ldots, n$; a measurable function, $f$, on $R^{n}$ is said to belong to $L^{P}=L^{P}\left(\bar{R}^{n}\right)$ if the number obtained after taking successively the $p_{1}$ norm in $x_{1}, \ldots$, the $p_{n}$ norm in $x_{n}$, and in that order, is finite. We denote this norm on $f$ by $\|f\|_{P}$. In particular, if $n=2$ and $1 \leq p_{i}<\infty$,

$$
\|f\|_{P}=\left\{\int_{\infty}^{\infty}\left\{\int_{\infty}^{\infty}\left|f\left(x_{1}, x_{2}\right)\right|^{p_{1}} d x_{1}\right\}^{p_{2} / p_{1}} d x_{2}\right\}^{1 / p_{1}}
$$

For the basic facts concerning these spaces see [3].

Note that $L^{P}$ can be regarded as $L^{p_{n}}$ of $L^{\bar{P}}$ valued functions where $\bar{P}=$ $\left(p_{1}, \ldots, p_{n-1}\right)$.

Suppose $k$ is a distribution in $\Lambda_{\alpha}^{1}, \alpha>0$, and $\psi$ is in $S_{0}$. Then clearly $k * \psi$ is well defined. It is also true that $k * f$ is well defined for $f$ in $L^{P}$. To see that this is so, choose $s, 0<s<\infty$, and write

$$
k=k^{(s)}+k_{(s)}
$$

where $k^{(s)}=\int_{0}^{s} k * \phi_{t} d t / t$ and $k_{(s)}=\int_{s}^{\infty} k * \phi_{t} d t / t$. Now $k^{(s)}$ is in $L^{1}$ and $k^{(s)}$ is in $L^{Q}$ whenever $Q=\left(q_{1}, \ldots, q_{n}\right)$ and $\sum_{i=1}^{n}\left(1-1 / q_{i}\right)>\alpha$. Hence, $k * f=$ $k^{(s)} * f+k_{(s)} * f$ is well defined for $f$ in $L^{P}$ whenever $P=\left(p_{1}, \ldots, p_{n}\right)$ and $\sum_{i=1}^{n}\left(1 / p_{i}\right)>\alpha$.

To see that $k^{(s)}$ is in $L^{1}$ write

$$
\left\|k^{(s)}\right\|_{1} \leq \int_{0}^{s}\left\|k * \phi_{t}\right\|_{1} \frac{d t}{t} \leq\|k\|_{1, \alpha} \int_{0}^{s} t^{\alpha-1} d t=\alpha^{-1}\|k\|_{1, \alpha} s^{\alpha}
$$


To see that $k_{(s)}$ is in $L^{Q}$, let $\psi$ be a function in $S_{0}$ so that $\hat{\psi}(\xi)=1$ whenever $\xi$ is in the support of $\phi$. Then $\phi_{t}=\phi_{t} * \psi_{t}$ and

$$
\left\|k_{(s)}\right\|_{Q} \leq \int_{s}^{\infty}\left\|k * \phi_{t}\right\|_{1}\left\|\psi_{t}\right\|_{Q} \frac{d t}{t} \leq\|k\|_{1, \alpha}\|\psi\|_{Q} \int_{s}^{\infty} t^{\alpha-\beta-1} d t
$$

where $\beta=\sum_{i=1}^{n}\left(1-1 / q_{i}\right)$. Since $\beta>\alpha$ the last integral coverges and the desired result follows.

TheOREM 3. Suppose $k$ is in $\Lambda_{\alpha}^{1}, \alpha>0$, and suppose $P=\left(p_{1}, \ldots, p_{n}\right)$ and $Q=\left(q_{1}, \ldots, q_{n}\right)$ satisfy $0 \leq 1 / q_{i} \leq 1 / p_{i} \leq 1, i=1, \ldots, n-1,0<1 / q_{n}<$ $1 / p_{n}<1$, and $\sum_{i=1}^{n}\left(1 / p_{i}-1 / q_{i}\right)=\alpha$. If $f$ is in $L^{P}$, then $k * f$ is in $L^{Q}$ and $\|k * f\|_{Q} \leq C\|f\|_{P}$, where $C$ is a constant which depends only on $P, Q$, and $\|k\|_{1, \alpha}$.

The conditions $p_{n}>1$ and $q_{n}<\infty$ cannot be relaxed. This can be easily seen by considering the case $p_{1}=\cdots=p_{n}, q_{1}=\cdots=q_{n}$, and taking $k$ to be the Riesz potential of order $\alpha$. However when $k$ is the Riesz potential of order $\alpha$ the results of [1] show that the conclusion of Theorem 3 holds in the case $1<p_{n}=q_{n}<\infty$. We do not know whether this is true in general. Nevertheless we can show that it does hold under further restrictions on $p_{n}$. These conditions are rather cumbersome. The following theorems contain the precise statements concerning this situation in the case $n=2$.

If $P=\left(p_{1}, p_{2}\right)$, we write $L^{p_{2}}\left(L^{p_{1}}\right)=L^{P}$ and \|\|$f\left\|_{p_{1}}\right\|_{p_{2}}=\|f\|_{P}$. The symbol $L^{p, q}$ denotes the usual Lorentz space of functions, see [1], and $L^{p_{2}}\left(L^{p_{1}, q_{1}}\right)$ denotes the class of functions $f$ which are measurable on $R^{2}$ and such that the norm

$$
\|\| f\left\|_{\left(p_{1}, q_{1}\right)}\right\|_{p_{2}}=\|\| f\left(x_{1} y\right)\left\|_{L^{p_{1}, q_{1}} \text { norm in } x}\right\|_{L^{p_{2}} \text { norm in } y}
$$

is finite.

We remind the reader that, for the sake of simplicity, the next theorems are stated only in the case $n=2$.

THEOREM 4. Suppose $k$ is in $\Lambda_{\alpha}^{1}, \alpha>0$, and $\left(p_{1}, p_{2}\right),\left(q_{1}, p_{2}\right)$ satisfy $1 / p_{1}-$ $1 / q_{1}=\alpha, 1<p_{1}<q_{1}<\infty$, and $1 \leq p_{2} \leq \infty$.

(i) If $p_{1} \leq p_{2} \leq \infty$ and $f$ is in $L^{p_{2}}\left(L^{p_{1}}\right)$ then $k * f$ is in $L^{p_{2}}\left(L^{q_{1}, p_{2}}\right)$ and

$$
\|\| k * f\left\|_{\left(q_{1}, p_{2}\right)}\right\|_{p_{2}} \leq C\|\| f\left\|_{p_{1}}\right\|_{p_{2}} \text {. }
$$

(ii) If $q_{1} \geq p_{2} \geq 1$ and $f$ is in $L^{p_{2}}\left(L^{p_{1}, p_{2}}\right)$ then $k * f$ is in $L^{p_{2}}\left(L^{p_{1}}\right)$ and

$$
\|\| k * f\left\|_{p_{1}}\right\|_{p_{2}} \leq C\|\| f\left\|_{\left(p_{1}, p_{2}\right)}\right\|_{p_{2}} .
$$

In both cases $C$ is a constant independent of $f$.

COROLLARY. With the same hypothesis as above and $p_{1} \leq p_{2} \leq q_{1}$ then the mapping $f \rightarrow k * f$ maps $L^{p_{2}}\left(L^{p_{1}}\right)$ continously into $L^{p_{2}}\left(L^{q_{1}}\right)$.

The restrictions on $p_{2}$ in the corollary cannot be removed by applying the methods of real interpolation to Theorem 4, see [4]. Concerning certain iterated variants of the real method see [6]. It should also be mentioned that it is not difficult to show that $f \rightarrow k * f$ maps $L^{P}$ into $B M O$ if $\sum_{i=1}^{n}\left(1 / p_{i}\right)=\alpha$. However this fact does not appear to be helpful in improving the range of $p_{2}$ in the corollary 
4. Details. Let $P$ and $Q$ satisfy the hypothesis of Theorem 3 and set $\bar{P}=$ $\left(p_{1}, \ldots, p_{n-1}\right), \bar{Q}=\left(q_{1}, \ldots, q_{n-1}\right)$. Regard $L^{P}$ as the class of $p_{n}$ summable strongly measurable $L^{\bar{P}}$ valued functions on $R$ and write $L^{P}=L^{p_{n}}\left(L^{\bar{P}}\right)$. Similarly write $L^{Q}=L^{q_{n}}\left(L^{\bar{Q}}\right)$. The proof of Theorem 3 is a vector valued analogue of the corresponding proof in [9]. That is, if $k$ is in $\Lambda_{\alpha}^{1}$ then the mapping $f \rightarrow k * f$ is of weak type $\left(p_{n}, q_{n}\right)$ in the following sense:

$$
\left|\left\{x_{n}:\left\|k * f\left(\bar{x}, x_{n}\right)\right\|_{\bar{Q}}>t\right\}\right| \leq C\left(t^{-1}\|f\|_{P}\right)^{q_{n}}
$$

where $C$ is a constant independent of $f,\|\|_{\bar{P}}$ denotes the $L^{\bar{P}}$ norm in the variable $\bar{x}=\left(x_{1}, \ldots, x_{n-1}\right)$, and $\mid$ set $\mid$ denotes the one dimensional Lebesgue measure of the set. The vector valued variant of the interpolation theorem of Marcinkiewicz, see [2], then implies the desired result.

To see (7) observe that it suffices to show that

$$
A_{s}(t)+B_{s}(t) \leq C t^{-q_{n}}
$$

whenever $f$ satisfies $\|f\|_{P}=1$, and

$$
\begin{aligned}
& A_{s}(t)=\left|\left\{x_{n}:\left\|k^{(s)} * f\left(\bar{x}, x_{n}\right)\right\|_{\bar{Q}}>t / 2\right\}\right|, \\
& B_{s}(t)=\left|\left\{x_{n}: \|\left. k_{(s)} * f\left(\bar{x}, x_{n}\right)\right|_{\bar{Q}}>t / 2\right\}\right|
\end{aligned}
$$

where $k^{(s)}$ and $k_{(s)}$ are defined by (4). Now

$$
A_{s}(t) \leq\left((2 / t)\|\| k^{(s)} * f\left(\bar{x}, x_{n}\right)\left\|_{\bar{Q}}\right\|_{p_{n}}\right)^{p_{n}},
$$

and if $\psi$ is the function used in deriving (6) we have

$$
\begin{aligned}
\|\| k^{(s)} * f\left(\bar{x}, x_{n}\right)\left\|_{\bar{Q}}\right\|_{p_{n}} & \leq \int_{0}^{s}\left\|k * \phi_{\tau}\right\|_{1}\left\|\psi_{\tau}\right\|_{\bar{r}}\|f\|_{P} \frac{d \tau}{\tau} \\
& \leq\|k\|_{1, \alpha} \int_{0}^{s} \tau^{\alpha-\beta-1} \frac{d \tau}{\tau}
\end{aligned}
$$

where $\bar{r}=\left(r_{1}, \ldots, r_{n-1}, 1\right), 1 / r_{i}=1+1 / q_{i}-1 / p_{i}$, and

$$
\beta=\sum_{i=1}^{n-1}\left(1-\frac{1}{r_{i}}\right)=\alpha-\frac{1}{p_{n}}+\frac{1}{q_{n}}<\alpha .
$$

Therefore

$$
A_{s}(t) \leq C_{1}\left(s^{\alpha-\beta} / t\right)^{p_{n}}
$$

Similarly

$$
\begin{aligned}
\|\| k_{(s)} * f\left(\bar{x}, x_{n}\right)\left\|_{\bar{Q}}\right\|_{\infty} & \leq \int_{s}\left\|k * \phi_{\tau}\right\|_{1}\left\|\psi_{\tau}\right\|_{\tilde{r}}\|f\|_{P} \frac{d \tau}{t} \\
& \leq\|k\|_{1, \alpha} \int_{s}^{\infty} \tau^{\alpha-\gamma-1} d t
\end{aligned}
$$

where $\tilde{r}=\left(r_{1}, \ldots, r_{n-1}, r_{n}\right), 1 / r_{i}=1+1 / q_{i}-1 / p_{i}, i=1, \ldots, n-1,1 / r_{n}=$ $1-1 / p_{n}$ and $\gamma=1 / p_{n}=\sum_{i=1}^{n-1}\left(1-1 / r_{i}\right)>\alpha$. Therefore,

$$
\|\| k_{(s)} * f\left(\bar{x}, x_{n}\right)\left\|_{\bar{Q}}\right\|_{\infty} \leq C_{2} s^{\alpha-\gamma} \text {. }
$$

Now choose $s$ so that the right-hand side of $(10)$ is equal to $t / 2$, and so $B_{s}(t)=0$. Using this value of $s$ in (9) we get

$$
A_{s}(t) \leq C t^{-q_{n}}
$$


and the desired result follows.

We now proceed to the proof of Theorem 4 .

Suppose that the transformation $f \rightarrow k * f$ maps $L^{\infty}\left(L^{p}\right)$ into $L^{\infty}\left(L^{q, \infty}\right)$ for $1 / q=1 / p-\alpha$ and $1<p<q<\infty$, that is

$$
\|\| k * f\left\|_{(q, \infty)}\right\|_{\infty} \leq C\|\| f\left\|_{p}\right\|_{\infty},
$$

where $C$ is a constant independent of $f$. By duality and the fact that this mapping is "selfadjoint" it follows that this transformation maps $L^{1}\left(L^{p, 1}\right)$ continuously into $L^{1}\left(L^{q}\right)$ for the same range of $p$ 's and $q$ 's. Applying the variant of the real method of interpolation which is valid when dealing with Lebesgue spaces of Banach space valued functions, see [4], it follows that $f \rightarrow k * f$ maps $L^{p_{2}}\left(L^{p, p_{2}}\right)$ into $L^{p_{2}}\left(L^{q, p_{2}}\right)$ if $1<p_{2}<\infty$. Since $L^{p_{2}}\left(L^{p}\right) \subset L^{p_{2}}\left(L^{p, p_{2}}\right)$ if $p \leq p_{2}$ and $L^{p_{2}}\left(L^{q, p_{2}}\right) \subset L^{p_{2}}\left(L^{q}\right)$ if $p_{2} \leq q$, Theorem 4 and the corollary follow.

To see (11), observe that, without loss of generality, one can take \|\|$f\left\|_{p}\right\|_{\infty}=1$, do, and write, for fixed $y$,

$$
F(t, y)^{1 / q} \leq A_{s}(t, y)^{1 / q}+B_{s}(t, y)^{1 / q},
$$

where we use $(x, y)$ to denote a point in $R^{2}$,

$$
\begin{aligned}
F(t, y) & =|\{x:|k * f(x, y)|>t\}|, \\
A_{s}(t, y) & =\left|\left\{x: k^{(s)} * f(x, y) \mid>t / 2\right\}\right|, \\
B_{s}(t, y) & =\left|\left\{x:\left|k_{(s)} * f(x, y)\right|>t / 2\right\}\right|
\end{aligned}
$$

and

Now

$$
k^{(s)}=\int_{0}^{s} k * \phi_{\tau} \frac{d \tau}{\tau}, \quad k_{(s)}=\int_{s}^{\infty} k * \phi_{\tau} \frac{d \tau}{\tau}
$$

$$
A(t, y)^{1 / p} \leq(2 / t)\left\|k^{(s)} * f(x, y)\right\|_{L^{p}(x)}
$$

and

$$
\left\|k^{(s)} * f(x, y)\right\|_{L^{p}(x)} \leq\left\|k^{(s)}\right\|_{1}\|\| f\left\|_{p}\right\|_{\infty} \leq C_{1} s^{\alpha} .
$$

Hence if $s=t^{\beta}$ where $\beta=(p-q) / \alpha p$ we have

$$
\|\| t\left(A_{s}(t, y)\right)^{1 / q}\left\|_{\infty}^{*}\right\|_{\infty} \leq C_{2}
$$

where \|\|$_{\infty}^{*}$ denotes the $L^{\infty}$ norm over $0<t<\infty$. To estimate $B_{s}$, take $r<\infty$ such that $1 / r=1 / u+1 / p-1,1-1 / u>\alpha$, and write

$$
B_{s}(t, y)^{1 / r} \leq(2 / t)\left\|k_{(s)} * f(x, y)\right\|_{L^{r}(x)},
$$

and

$$
\left\|k_{(s)} * f(x, y)\right\|_{L^{r}(x)} \leq\|\| k_{s}\left\|_{u}\right\|_{1}\|\| f\left\|_{p}\right\|_{\infty} \leq C_{3} s^{\alpha-\gamma}
$$

where $\gamma=1-1 / u$. Since $s=t^{\beta}, \beta=(p-q) / \alpha p, s^{\alpha-\gamma}=t^{1-(q / r)}$. Hence

$$
\|\| t\left(B_{s}(t, y)\right)^{1 / q}\left\|_{\infty}^{*}\right\|_{\infty} \leq C_{4} .
$$

Inequalities (12), (13), and (14) imply

$$
\|\| t(F(t, y))^{1 / q}\left\|_{\infty}^{*}\right\|_{\infty} \leq C_{4}
$$

which is the desired result. 


\section{REFERENCES}

1. D. R. Adams and R. J. Bagby, Translation-dilation invariant estimates for Riesz potentials, Indiana Univ. Mat. J. 23 (1974) 1051-1067.

2. A. Benedek, A. P. Calderon and R. Panzone, Convolution operators on Banach space valued functions, Proc. Nat. Acad. Sci. U.S.A. 48 (1962), 356-365.

3. A. Benedek and R. Panzone, The spaces $L^{p}$ with mixed norm, Duke Math. J. 28 (1961), 301324.

4. M. Cwikel, On $\left(L^{p_{0}}\left(A_{0}\right), L^{p_{1}}\left(A_{1}\right)\right)_{\theta q}$, Proc. Amer. Math. Soc. 44 (1974), 286-292.

5. P. I. Lizorkin, Multipliers of Fourier integrals and bounds of convolutions in spaces with mixed norms, Izv. Akad. Nauk SSSR 4 (1970), 225-254.

6. M. Milman, On interpolation of $2^{n}$ Banach spaces and Lorentz spaces with mixed norms, J. Funct. Anal. 11 (1981), 1-7.

7. J. Peetre, New thoughts on Besov spaces, Math. Dept., Duke Univ., Durham, N.C., 1976.

8. N. M. Riviere, The Fourier method in approximation theory, unpublished notes.

9. E. M. Stein and A. Zygmund, Boundedness of translation invariant operators on Hölder and $L^{p}$ spaces, Ann. of Math. (2) 85 (1967), 337-349.

10. M. H. Taibleson, On the theory of Lipschitz spaces of distributions on Euclidean $n$-space. II, Indiana Univ. Math. J. 14 (1965), 821-840.

11. A. Zygmund, On the preservation of classes of functions, J. Math. Mech. 8 (1959), 889-896.

Department of Mathematics, Iowa State University, Ames, Iowa 50011 